\title{
Prognostic Significance of the Expression of CD98 (4F2hc) in Gastric Cancer
}

\author{
TAISUKE SATOH ${ }^{1}$, KYOICHI KAIRA $^{2}$, KENGO TAKAHASHI $^{1}$, NORIHUMI TAKAHASHI ${ }^{1}$, \\ YOSHIKATSU KANAI ${ }^{3}$, TAKAYUKI ASAO ${ }^{4}$, JUN HORIGUCHI ${ }^{1}$ and TETSUNARI OYAMA ${ }^{5}$ \\ ${ }^{1}$ Department of Thoracic and Visceral Organ Surgery, \\ Gunma University Graduate School of Medicine, Gunma, Japan; \\ ${ }^{2}$ Department of Oncology Clinical Development, Gunma University Graduate School of Medicine, Gunma, Japan; \\ ${ }^{3}$ Division of Bio-system Pharmacology, Graduate School of Medicine, Osaka University, Osaka, Japan; \\ ${ }^{4}$ Big Data Center for Integrative Analysis, \\ Gunma University Initiative for Advance Research (GIAR), Gunma, Japan; \\ ${ }^{5}$ Department of Diagnostic Pathology, Gunma University Graduate School of Medicine, Gunma, Japan
}

\begin{abstract}
Background: $C D 98$ expression is high in various human neoplasms. However, the relationship of CD98 expression with the clinicopathological factors of gastric cancer (GC) remains unclear. This study examined CD98 expression and its clinicopathological impact on GC. Patients and Methods: Three hundred and thirty-one patients with surgically resected $G C$ were evaluated. Tumor sections were stained and analyzed using immunohistochemistry to assess CD98 expression. Results: CD98 was positively expressed in 19\% (66/331) of our patient cohort. Increased CD98 expression was significantly associated with advanced GC stage, lymph node metastasis, non-signet histology, lymphatic permeation, and vascular invasion. Positive CD98 expression was also a significant prediction marker for unfavorable prognosis postoperatively. However, CD98 was not identified as GC's independent prognostic predictor. Conclusion: CD98 could be a novel prediction marker for worse prognosis in GC-affected patients. Our data suggests that increased CD98 expression plays an essential role in tumor aggressiveness and metastasis.
\end{abstract}

Gastric cancer (GC) is the most common malignancy of gastrointestinal neoplasms. Recent improvement in survival after treatment is observed; however, patients with advanced

Correspondence to: Kyoichi Kaira, MD, Ph.D., Department of Oncology Clinical Development, Gunma University Graduate School of Medicine, Showa-machi, Maebashi, Gunma 371-8511, Japan. Tel: +81 27220-8136, Fax: +81 272208136, e-mail: kkaira1970@yahoo.co.jp

Key Words: Gastric cancer, CD98, amino acid transporter, immunohistochemistry, prognostic factor.
GC still have low survival despite standard chemotherapy. Moreover, no established biomarker can predict survival outcomes and responses after therapeutic modalities, such as surgery or systemic chemotherapy. Thus, finding a viable predictive biomarker in GC-affected patients is crucial. The CD98 family comprises of widely expressed cell surface heterodimer proteins composed of a glycosylated heavy and a non-glycosylated light chain. Particularly, CD98 is a disulfide-linked $125-\mathrm{kDa}$ heterodimeric membrane glycoprotein; it has been described to be associated with cellular proliferation, transformation, adhesion and the L-type amino acid transporter (LAT) system $(1,2)$. CD98 $(4 \mathrm{~F} 2 \mathrm{hc})$ expression is elevated in various human neoplasms, such as lung, pancreatic, biliary tract, breast, prostate, and head and neck cancers; hepatocellular carcinoma; and myeloma (3-12). Moreover, it has been reported that increased CD98 expression is significantly associated with shorter survival outcomes, tumor cell proliferation, and metastases (8). CD98 assists in transporting many amino acids in a cooperative function with L-type amino acid transporter 1 (LAT1) and $\mathrm{xCT}$ on the membranous surface of cancer cells (9). The LAT1 expression is elevated in various human cancers (2). We have previously reported that enhanced LAT1 expression is a significant prognostic marker correlated with tumor cell proliferation, angiogenesis, and CD98 expression level (3, 4, 8, 9). Recently, Ichinoe et al. described that LAT1 overexpression is closely associated with worse survival and lymph node metastases. However, since only 87 patients with GC were examined by immunohistochemistry against LAT1, this observation could be biased because of the small sample size. In the same study, LAT1 was also found as an effective predictor for non-scirrhous gastric cancer (13). Inhibition of LAT1 suppresses cancer cell growth via the mTOR signaling pathway (14). As a novel mechanism, LAT1 inhibition causes 
cell-cycle arrest at $\mathrm{G}_{1}$ phase and several studies reported apoptosis in some cell lines upon LAT1 inhibition $(15,16)$. Although CD98 expression levels are closely correlated with LAT1 expression, the mechanism of CD98 inhibition and tumor suppression in various human neoplasms remain unclear. Recent reports demonstrated CD98 as an attractive target for cancer treatment $(4,8)$. However, it remains unknown whether CD98 is associated with survival outcomes and clinicopathological factors in GC-affected patients. To bridge this gap in knowledge, we investigated the impact of CD98 expression on the pathological dynamics of GCaffected patients.

\section{Patients and Methods}

Patients. Under the retrospective design of the study herein, 331 patients were selected who underwent surgery for GC at the Gunma University Hospital between January 2000 and December 2009. All clinicopathological data such as age, gender, histology, lymphatic permeation, vascular invasion, lymph node metastasis, and disease staging, were obtained from the institution database. Data were retrospectively analyzed. The Institutional Review Board of Gunma University Hospital (Gunma University Faculty of Medicine's Ethical Committee for Clinical Studies) approved this study. Patients' age ranged from 28 to 90 years, and the median age was 69 years. None of the patients had received neo-adjuvant chemotherapy. All surgical specimens were reviewed and classified according to the WHO classification by an experienced pathologist, blinded to clinical or imaging findings. Pathological tumor-nodemetastasis stages were established using the International System for Staging adopted by the American Joint Committee on Cancer and the Union Internationale Contre le Cancer. In our analysis, the clinicopathological factors such as tumor differentiation, lymphatic permeation and vascular invasion were used. In terms of histology, all patients had adenocarcinoma (AC); 181, 66, 64, and 20 patients had stage I, II, III, and IV tumors, respectively. The day of surgery was considered the starting day for measuring postoperative survival. The follow-up duration ranged from 72 to 5,430 days (median, 2,047 days). As a comorbid disease in our study, 125 patients were treated with a medication for hypertension. The author's approach for the evaluation and resection of these tumors has been previously described (17).

Immunohistochemical staining. CD98 expression was determined by immunohistochemical staining using an anti-CD98 antibody. The anti-CD98 antibody is an affinity purified rabbit polyclonal antibody (Santa Cruz Biotechnology, Inc, Dallas, TX, U.S.A. 1:100 dilution) raised against a peptide mapping at the $\mathrm{C}$-terminus of human $\mathrm{CD} 98$ (3, 4, 10-12). Immunohistochemical staining was performed on paraffin sections using a polymer peroxidase method (Histofine Simple Stain MAX PO (MULTI) kit; Nichirei Corporation, Tokyo, Japan). Deparaffinized, rehydrated sections were briefly treated with $0.3 \%$ hydrogen peroxidase in methanol for $30 \mathrm{~min}$ to block endogenous peroxidase activity. After rinsing in phosphate-buffered saline, the sections were incubated with the anti-CD98 antibody $(1: 100)$ overnight. Thereafter, sections were incubated with the Histofine Simple Stain MAX PO (MULTI) kit (Nichirei Corporation). The peroxidase reaction was performed using $0.02 \%$ 3,3'-diaminobenzidine tetrahydrochloride and $0.01 \%$ hydrogen peroxidase in $0.05 \mathrm{M}$ tris- $\mathrm{HCl}$ buffer, $\mathrm{pH}$ 7.6. Negative control tissue sections were prepared by omitting the primary antibody. CD98 expression was considered to be positive only if plasma membrane staining was presen and it was scored by assessing the extent of staining as follows: score $=1, \leq 10 \%$ of tumor area stained; score $=2,11 \%-25 \%$ stained; score $=3,26 \%-50 \%$ stained; and score $=4, \geq 51 \%$ stained. Tumors with scores $\geq 2$ were defined as CD98 positive. The sections were assessed using light microscopy by at least two authors who were blinded to the results.

Statistical analysis. p-Values of $<0.05$ indicated statistically significant difference. The significance of difference was determined by Fisher's exact test. The Kaplan-Meier method was used to estimate survival as a function of time and survival differences were analyzed using the log-rank test. Overall survival (OS) was defined as the time from tumor resection to death from any cause. Progression-free survival (PFS) was defined as the time between tumor resection and the initial manifestation of disease progression or death. Multivariate analyses were performed using the stepwise Cox proportional hazards model to identify independent prognostic factors. Statistical analyses were performed using GraphPad Prism 4 (Graph Pad Software, San Diego, CA, USA) and JMP 8 (SAS, Institute Inc., Cary, NC, USA) for Windows.

\section{Results}

Patient demographics and immunohistochemical analyses. The immunohistochemical analyses were performed on 331 primary GC lesions. CD98 immunostaining was predominantly localized on the plasma membrane (Figure 1). In our patient cohort, the positive expression rate of CD98 was $19 \%$ (66/331). Figure 2 shows the CD98 scoring profiles, in which scores of $1,2,3$, and 4 were observed in $80 \%, 12 \%, 7 \%$, and $1 \%$ of GC lesions, respectively. Patient demographics according to CD98 expression status are listed in Table I. CD98 expression was significantly associated with disease stage, $\mathrm{T}$ factor, lymph node metastases, nonsignet cells, lymphatic permeation, and vascular invasion.

Survival analysis based on CD98 expression. The 5-year OS and PFS rate of all patients was $75 \%$ and $74 \%$, respectively. Out of 331 patients, 102 died after the initial surgery. Using univariate analysis, it was found that age, disease stage, $\mathrm{T}$ factor, $\mathrm{N}$ factor, lymphatic permeation, vascular invasion and CD98 expression had a significant relationship with OS. Meanwhile, disease stage, $\mathrm{T}$ factor, $\mathrm{N}$ factor, lymphatic permeation and vascular invasion were closely related to unfavorable PFS (Table II). Multivariable analysis confirmed that disease stage, age and $\mathrm{T}$ factor were independent prognostic factors for predicting poor OS and that disease stage, $\mathrm{T}$ factor and vascular invasion were significant predictive factors for PFS in GC-affected patients. Figures $3 \mathrm{~A}$ and $3 \mathrm{~B}$ illustrate the Kaplan-Meier survival curve in patients with high and low CD98 expression, respectively. The prognostic significance of CD98 expression was 

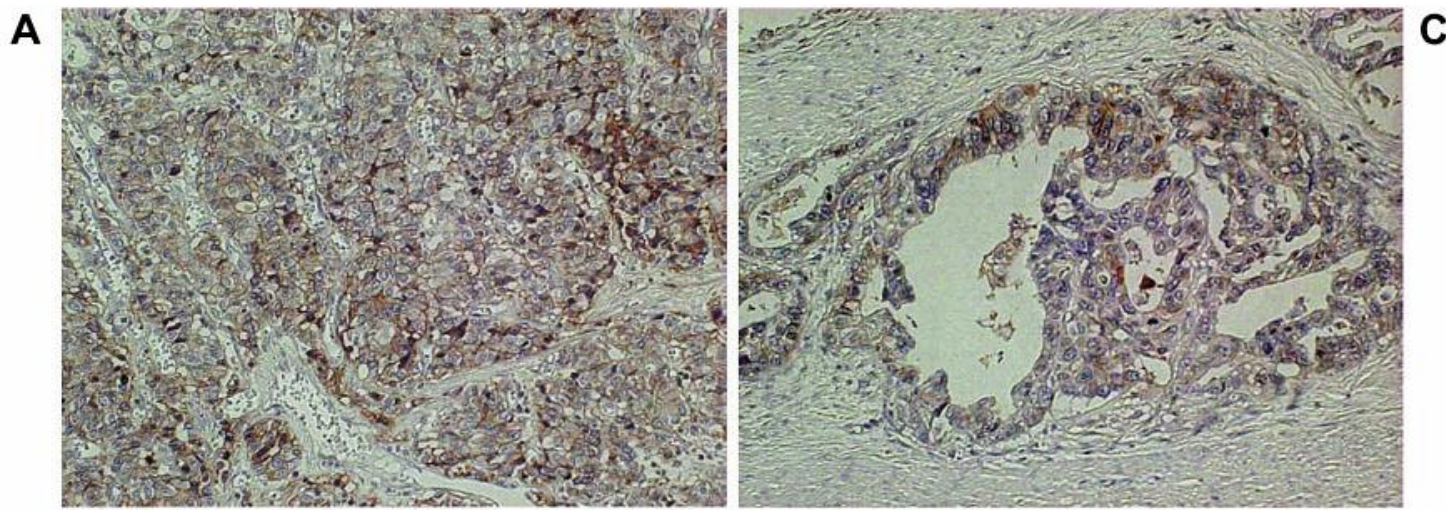

B
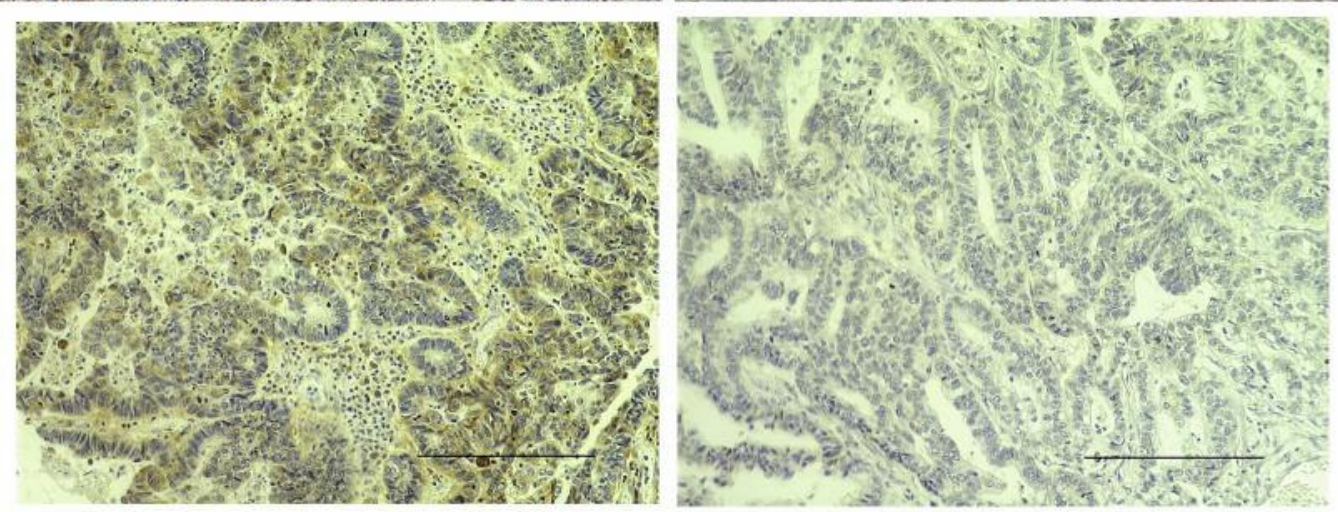

\section{D}

Figure 1. Immunohistochemical staining of CD98 in gastric adenocarcinoma. Positive CD98 staining is localized to the membrane of a malignant melanoma. In the representative images, CD98 immunostaining was scored as grade $4(A), 3(B), 2(C)$ and grade 1 (D).

analyzed according to disease stage and tumor differentiation. No statistically significant difference in OS and PFS was observed between patients with either positive or negative CD98 expression in early (stage I or II) versus advanced stage disease (III or IV). However, the KaplanMeier analysis of OS demonstrated a significant difference between patients with either positive or negative CD98 expression and with well or moderate differentiation (Figure 3C), but no such significance was observed in patients with poor differentiation (Figure 3D).

\section{Discussion}

This study represents the first evaluation of the clinical and pathological significance of CD98 expression in patients with surgically resected GC. We found that increased CD98 expression was significantly associated with tumor aggressiveness and metastasis. CD98 is a potential biomarker that can be used to predict GC patient outcomes after surgical resection. Our study highlighted the prognostic significance of CD98 expression regarding GC-affected patients with well-to-moderate differentiation. These results suggested that $\mathrm{CD} 98$ plays a crucial role in tumor

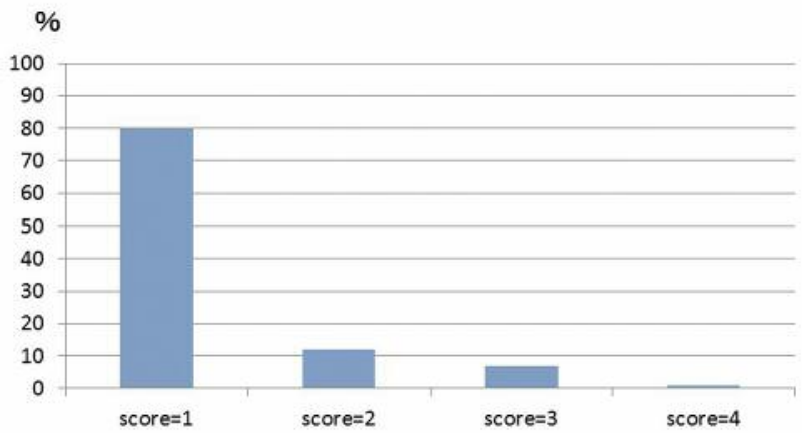

Figure 2. Scoring of CD98 expression in GC tumor sections. CD98 scores of 1, 2, 3, and 4 were observed in $80 \%, 12 \%, 7 \%$, and $1 \%$ of GC tumor sections, respectively.

progression and GC invasion. Because the mechanism governing such dynamics is unknown, further studies investigating a GC population without poor differentiation is warranted to verify CD98's clinical role in GC. While numerous studies have shown that increased CD98 expression is associated with clinical and pathological features in different cancers, the magnitude of positive CD98 

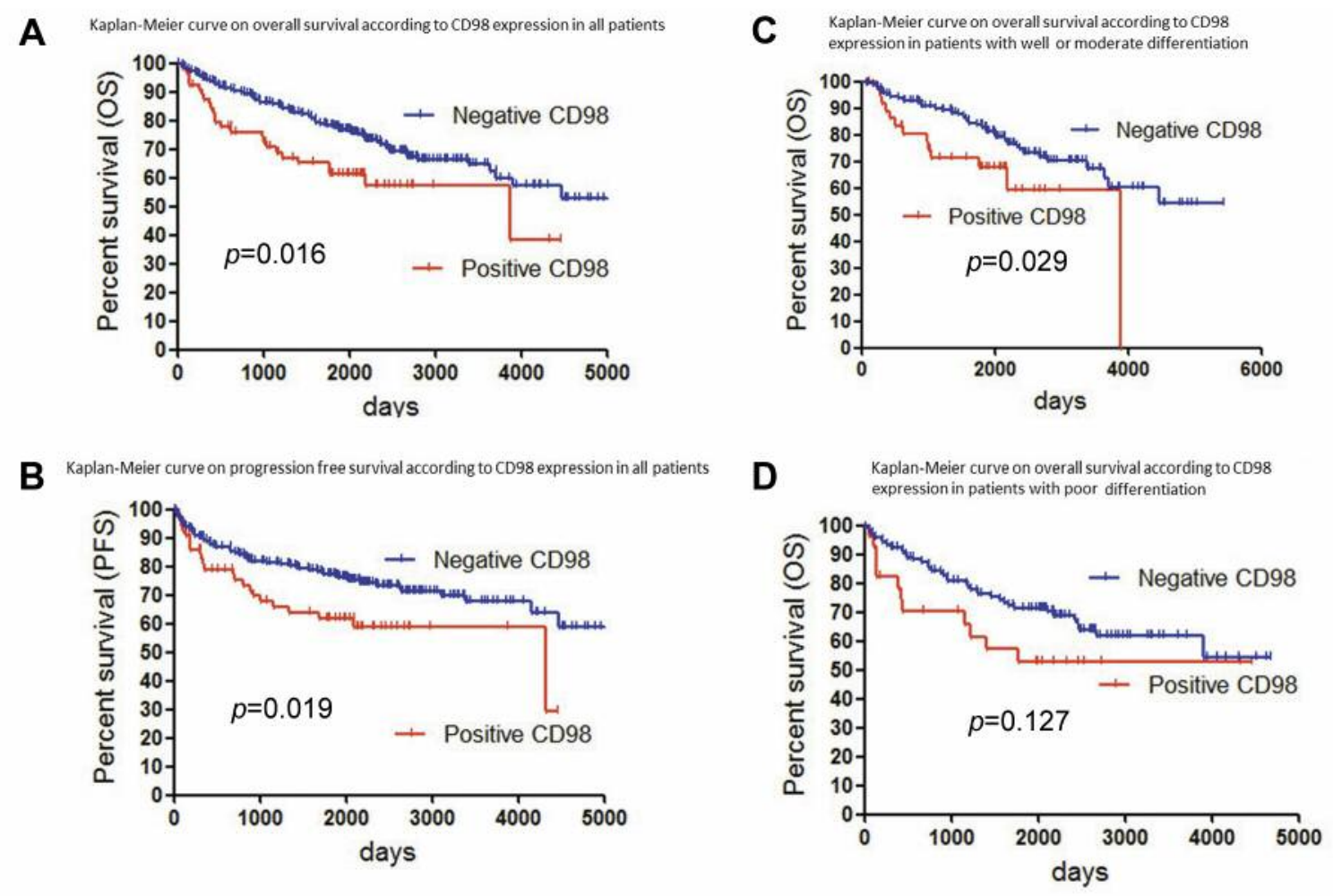

Figure 3. Survival outcomes after surgical resection. Kaplan-Meier analysis was conducted to assess the impact of CD98 expression on overall survival (OS) and progression-free survival (PFS) in GC-affected patients. A statistically significant difference in OS (A) and PFS (B) was observed between patients with positive or negative CD98 expression. Additionally, Kaplan-Meier analysis of OS resulted in a significant difference between patients with positive or negative CD98 expression and with either well or moderate differentiation (C), but there was no observed significant difference in patients with poor differentiation $(D)$.

expression (19\%) in our GC patient cohort seems to be markedly lower in comparison. The positive rates of CD98 expression in lung, pancreatic, biliary tract, breast, and hypopharyngeal cancers are $47 \%, 56.7 \%, 36.7 \%, 31.8 \%$, and $82.3 \%$, respectively $(3-5,8-10)$. As mentioned above, CD98 expression levels correlate with LAT1 expression levels (35). Our group, has previously noticed that LAT1 expression is higher in patients with squamous cell carcinoma (SQC) than in those with AC (7). Moreover, in patients with lung cancer, LAT1 expression in SQC (with a positive rate of 91\%) was significantly higher than in AC (with a positive rate of 29\%). The prognostic significance of LAT1 expression has also been shown to be markedly different between patients with SQC and AC. Particularly, LAT1 expression in patients with $\mathrm{AC}$ has a direct relationship with worse prognosis, whereas there is a weak association between LAT1 expression and survival in SQC-affected patients. In patients with early stage cancer, LAT1 expression associated more with shorter survival compared to CD98 expression. However, CD98's prognostic role may be more important than that of LAT1 in advanced-stage cancer progression. Additionally, it is uncertain whether the
Table I. Patient's demographics according to CD98 expression.

\begin{tabular}{|c|c|c|c|c|}
\hline \multirow[t]{2}{*}{ Variables } & \multirow{2}{*}{$\begin{array}{c}\text { Total } \\
(\mathrm{n}=331)\end{array}$} & \multicolumn{2}{|c|}{ CD98 expression } & \multirow[t]{2}{*}{$p$-Value } \\
\hline & & $\begin{array}{c}\text { Positive } \\
(\mathrm{n}=66)\end{array}$ & $\begin{array}{c}\text { Negative } \\
(n=265)\end{array}$ & \\
\hline \multicolumn{5}{|l|}{ Age } \\
\hline$<65$ years $/ \geq 65$ years & $132 / 199$ & $20 / 46$ & $112 / 153$ & 0.09 \\
\hline \multicolumn{5}{|l|}{ Gender } \\
\hline Male/Female & $228 / 103$ & $50 / 16$ & $178 / 87$ & 0.23 \\
\hline \multicolumn{5}{|l|}{ Disease stage } \\
\hline I or II/III or IV & $247 / 84$ & $36 / 30$ & $211 / 54$ & $<0.01$ \\
\hline \multicolumn{5}{|l|}{$\mathrm{T}$ factor } \\
\hline T1-2/ T3-4 & $208 / 123$ & $26 / 40$ & $182 / 83$ & $<0.01$ \\
\hline \multicolumn{5}{|l|}{$\mathrm{N}$ factor } \\
\hline N0/N1-3 & $216 / 115$ & $30 / 36$ & $186 / 79$ & $<0.01$ \\
\hline \multicolumn{5}{|l|}{ Differentiation } \\
\hline Well or moderate/poorly & $183 / 148$ & $37 / 29$ & 146/109 & 0.89 \\
\hline \multicolumn{5}{|l|}{ Histology } \\
\hline Signet/Non-signet & $40 / 291$ & $0 / 66$ & $40 / 225$ & $<0.01$ \\
\hline \multicolumn{5}{|l|}{ Lymphatic permeation } \\
\hline Positive/Negative & $188 / 143$ & $53 / 13$ & $135 / 130$ & $<0.01$ \\
\hline \multicolumn{5}{|l|}{ Vascular invasion } \\
\hline Positive/Negative & $128 / 203$ & $46 / 20$ & $82 / 183$ & $<0.01$ \\
\hline
\end{tabular}


Table II. Univariate and multivariate survival analysis in all patients.

\begin{tabular}{|c|c|c|c|c|c|c|c|c|c|c|}
\hline \multirow[t]{3}{*}{ Variables } & \multicolumn{5}{|c|}{ Overall survival } & \multicolumn{5}{|c|}{ Disease-free survival } \\
\hline & \multicolumn{2}{|c|}{ Univariate } & \multicolumn{3}{|c|}{ Multivariate } & \multicolumn{2}{|c|}{ Univariate } & \multicolumn{3}{|c|}{ Multivariate } \\
\hline & 5 years $(\%)$ & $p$-Value & HR & $95 \% \mathrm{CI}$ & $p$-Value & 5 years $(\%)$ & $p$-Value & HR & $95 \% \mathrm{CI}$ & $p$-Value \\
\hline \multicolumn{11}{|l|}{ Age } \\
\hline$<65$ years $/ \geq 65$ years & $79 / 72$ & 0.008 & 1.426 & $1.149-1.791$ & 0.001 & $78 / 74$ & 0.258 & & & \\
\hline \multicolumn{11}{|l|}{ Gender } \\
\hline Male/Female & $73 / 78$ & 0.334 & $74 / 78$ & 0.461 & & & & & & \\
\hline \multicolumn{11}{|l|}{ Disease stage } \\
\hline $\mathrm{I}$ or $\mathrm{II} / \mathrm{III}$ or IV & $85 / 43$ & $<0.001$ & 1.464 & $1.123-1.917$ & 0.005 & $87 / 42$ & $<0.001$ & 1.587 & $1.214-2.091$ & $<0.001$ \\
\hline \multicolumn{11}{|l|}{$\mathrm{T}$ factor } \\
\hline T1-2/T3-4 & $91 / 47$ & $<0.001$ & 1.678 & $1.274-2.235$ & $<0.001$ & $92 / 47$ & $<0.001$ & 1.779 & $1.300-2.481$ & $<0.001$ \\
\hline \multicolumn{11}{|l|}{$\mathrm{N}$ factor } \\
\hline N0/N1-3 & $85 / 54$ & $<0.001$ & 1.092 & $0.842-1.413$ & 0.501 & $86 / 56$ & $<0.001$ & 1.063 & $0.811-1.383$ & 0.652 \\
\hline \multicolumn{11}{|l|}{ Differentiation } \\
\hline Well or moderate/poorly & $81 / 68$ & 0.059 & $80 / 69$ & 0.054 & & & & & & \\
\hline \multicolumn{11}{|l|}{ Histology } \\
\hline Signet/Non-signet & $83 / 74$ & 0.071 & $83 / 75$ & 0.05 & & & & & & \\
\hline \multicolumn{11}{|l|}{ Lymphatic permeation } \\
\hline Positive/Negative & $62 / 93$ & $<0.001$ & 1.303 & $0.927-1.838$ & 0.127 & $63 / 91$ & $<0.001$ & 1.146 & $0.779-1.691$ & 0.486 \\
\hline \multicolumn{11}{|l|}{ Vascular invasion } \\
\hline Positive/Negative & $50 / 90$ & $<0.001$ & 1.231 & $0.943-1.623$ & 0.127 & $51 / 90$ & $<0.001$ & 1.451 & $1.091-1.971$ & 0.009 \\
\hline \multicolumn{11}{|l|}{ CD98 } \\
\hline Positive/Negative & $62 / 78$ & 0.016 & 0.992 & $0.772-1.254$ & 0.948 & $63 / 78$ & 0.019 & 1.115 & $0.876-1.440$ & 0.38 \\
\hline
\end{tabular}

prognostic roles of LAT1 and CD98 are different between patients with early and advanced stages and this warrants rigorous investigation.

Recently, Toyoda et al. investigated the prognostic significance of LAT1 and CD98 in patients with advanced hypopharyngeal SQC and concluded that CD98 and not LAT1, could serve as a prognostic marker to predict survival outcomes postoperatively, although LAT1 and CD98 were positively expressed at $85.7 \%$ and $82.8 \%$ of the samples studied, respectively. Additionally, this study exhibited a statistically significant correlation between the expression of LAT1 and CD98. Although CD98's role as a potentially promising and predictive biomarker in advanced human neoplasms $(4,8)$ is important, there was no significant difference between patients with GC either positive or negative for CD98 expression in terms of advanced stage. Interestingly, our data demonstrated that a significantly worse prognosis was confirmed in GCaffected patients with positive CD98 expression. As stated earlier, Ichinoe et al. noted that LAT1 could be an independent prognostic factor in GC-affected patients without scirrhous carcinoma (13). As scirrhous carcinoma is associated with poor differentiation, LAT1 may be a significant prognostic predictor for GC-affected patients without poor differentiation. Such dynamics are somewhat reflective in our data.
Our study has several limitations. First, we did not investigate the relationship between CD98 expression and the integrin signaling pathway and teasing out this relationship merits future studies. Second, a heterogeneous patient population was evaluated, and such an analysis may bias the results of survival outcomes postoperatively. Next, steps will involve studying survivorship in a homogenous GC patient population. In conclusion, CD98 was positively expressed in $19 \%$ of GC-affected patients who underwent surgery and CD98 expression was significantly associated with GC tumor aggression and metastases. CD98 predicted worse prognosis in GCaffected patients, particularly in patients without poor differentiation. However, this result was not confirmed using multivariable analysis. CD98 could be an attractive target for GC therapeutic treatments.

\section{Acknowledgements}

The Authors would like to thank Ms. Yuka Matsui for her technical assistance and deeply appreciate Prof. Masahiko Nishiyama of the Department of Molecular Pharmacology and Oncology, Gunma University Graduate School of Medicine for critical reviewing this manuscript. The Authors declare that they have no conflict of interest. 


\section{References}

1 Cantor JM and Ginsberg MH: CD98 at the crossroads of adaptive immunity and cancer. J Cell Sci 125: 1373-1382, 2012.

2 Kanai Y, Segawa H, Miyamoto K, Uchino H, Takeda E and Endou $\mathrm{H}$ : Expression cloning and characterization of a transporter for large neutral amino acids activated by the heavy chain of 4F2 antigen (CD98). J Biol Chem 273: 23629-23632, 1998.

3 Kaira K, Sunose Y, Arakawa K, Ogawa T, Sunaga N, Shimizu $\mathrm{K}$, Tominaga $\mathrm{H}$, Oriuchi $\mathrm{N}$, Itoh $\mathrm{H}$, Nagamori S, Kanai $\mathrm{Y}$, Segawa A, Furuya M, Mori M, Oyama T and Takeyoshi I: Prognostic significance of L-type amino-acid transporter 1 expression in surgically resected pancreatic cancer. Br J Cancer 107: 632-638, 2012.

4 Kaira K, Oriuchi N, Imai H, Shimizu K, Yanagitani N, Sunaga N, Hisada T, Kawashima O, Kamide Y, Ishizuka T, Kanai Y, Nakajima T and Mori M: CD98 expression is associated with poor prognosis in resected non-small-cell lung cancer with lymph node metastases. Ann Surg Oncol 16: 3473-3481, 2009.

5 Furuya M, Horiguchi J, Nakajima H, Kanai Y and Oyama T: Correlation of L-type amino acid transporter 1 and CD98 expression with triple negative breast cancer prognosis. Cancer Sci 103: 382-389, 2012.

6 Sakata T, Ferdous G, Tsuruta T, Satoh T, Baba S, Muto T, Ueno A, Kanai Y, Endou $\mathrm{H}$ and Okayasu I: L-type amino acid transporter 1 as a novel biomarker for high-grade malignancy in prostate cancer. Pathol Int 59: 7-18, 2009.

7 Kaira K, Oriuchi N, Imai H, Shimizu K, Yanagitani N, Sunaga N, Hisada T, Tanaka S, Ishizuka T, Kanai Y, Endou H, Nakajima $\mathrm{T}$ and Mori M: Prognostic significance of L-type amino acid transporter 1 expression in resectable stage I-III nonsmall cell lung cancer. Br J Cancer 98: 742-748, 2008.

8 Toyoda M, Kaira K, Shino M, Sakakura K, Takahashi K, Takayasu Y, Tominaga H, Oriuchi N, Nikkuni O, Suzuki M, Iijima M, Tsukamoto N, Nagamori S, Kanai Y, Oyama T and Chikamatsu K: CD98 is a novel prognostic indicator for patients with stage III/IV hypopharyngeal squamous cell carcinoma. Head Neck 37: 1569-1574, 2015.

9 Toyoda M, Kaira K, Ohshima Y, Ishioka NS, Shino M, Sakakura K, Takayasu Y, Takahashi K, Tominaga H, Oriuchi N, Nagamori S, Kanai Y, Oyama T and Chikamatsu K: Prognostic significance of amino acid transporter expression (LAT1, ASCT2 and xCT) in surgically resected tongue cancer. Br J Cancer 110: 2506$2513,2014$.

10 Kaira K, Sunose Y, Oriuchi N, Kanai Y and Takeyoshi I: CD98 as a promising biomarker of prognosis in biliary tract cancer. Hepatobiliary Pancreat Dis Int 13: 654-657, 2014.

11 Isoda A, Kaira K, Iwashina M, Oriuchi N, Tominaga H, Nagamori S, Kanai Y, Oyama T, Asao T, Matsumoto M and Sawamura M: Expression of L-type amino acid transporter 1 (LAT1) as a prognostic and therapeutic indicator in multiple myeloma. Cancer Sci 105: 1496-1502, 2014.
12 Namikawa M, Kakizaki S, Kaira K, Tojima H, Yamazaki Y, Horiguchi N, Sato K, Oriuchi N, Tominaga H, Sunose Y, Nagamori S, Kanai Y, Oyama T, Takeyoshi I and Yamada M: Expression of amino acid transporters (LAT1, ASCT2 and xCT) as clinical significance in hepatocellular carcinoma. Hepatol Res 45: 1014-1022, 2014.

13 Ichinoe M, Mikami T, Yoshida T, Igawa I, Tsuruta T, Nakada N, Anzai N, Suzuki Y, Endou H and Okayasu I: High expression of L-type amino-acid transporter 1 (LAT1) in gastric carcinomas: comparison with non-cancerous lesions. Pathol Int 61: 281-289, 2011.

14 Imai H, Kaira K, Oriuchi N, Shimizu K, Tominaga H, Yanagitani N, Sunaga N, Ishizuka T, Nagamori S, Promchan K, Nakajima T, Yamamoto N, Mori M and Kanai Y: Inhibition of L-type amino acid transporter 1 has antitumor activity in non-small cell lung cancer. Anticancer Res 30: 4819-4828, 2010.

$15 \mathrm{Kim}$ CS, Cho SH, Chun HS, Lee SY, Endou H, Kanai Y and Kim DK: BCH, an inhibitor of system $\mathrm{L}$ amino acid transporters, induces apoptosis in cancer cells. Biol Pharm Bull 31: 10961100,2008

16 Kim CS, Moon IS, Park JH, Shin WC, Chun HS, Lee SY, Kook JK, Kim HJ, Park JC, Endou H, Kanai Y, Lee BK and Kim DK: Inhibition of L-type amino acid transporter modulates the expression of cell cycle regulatory factors in $\mathrm{KB}$ oral cancer cells. Biol Pharma Bull 33: 1117-1121, 2010.

17 Takahashi K, Kaira K, Shimizu A, Sato T, Tamahashi N, Ogawa H, Yoshinari D, Yokobori T, Asao T, Takeyoshi I and Oyama T: Clinical significance of $\beta 2$-adrenergic receptor expression in patients with surgically resected gastric adenocarcinoma. Tumor Biol: 1-8, 2016.

18 Kaira K, Oriuchi N, Takahashi T, Nakagawa K, Ohde Y, Okumura T, Murakami H, Shukuya T, Kenmotsu H, Naito T, Kanai Y, Endo M, Kondo H, Nakajima $\mathrm{T}$ and Yamamoto N: LAT1 expression is closely associated with hypoxic markers and mTOR in resected non-small cell lung cancer. Am J Transl Res 3: 468-478, 2011.

19 Feral CC, Nishiya N, Fenczik CA, Stuhlmann H, Slepak M and Ginsberg MH: CD98hc (SLC3A2) mediates integrin signaling. Proc Natl Acad Sci USA 102: 355-360, 2005.

20 Yang Y, Toy W, Choong LY, Hou P, Ashktorab H, Smoot DT, Yeoh KG and Lim YP: Discovery of SLC3A2 Cell Membrane Protein as a Potential Gastric Cancer Biomarker: Implications in Molecular Imaging. J Proteome Res 11: 5736-5747, 2012. 